\title{
Correlated Sources Over Wireless Channels: Cooperative Source-Channel Coding
}

\author{
Arul D. Murugan, Praveen K. Gopala, and Hesham El Gamal, Member, IEEE
}

\begin{abstract}
We consider wireless sensor networks deployed to observe arbitrary random fields. The requirement is to reconstruct an estimate of the random field at a certain collector node. This creates a many-to-one data gathering wireless channel. One of the main challenges in this scenario is that the source/channel separation theorem, proved by Shannon for point-to-point links, does not hold anymore. In this paper, we construct novel cooperative source-channel coding schemes that exploit the wireless channel and the correlation between the sources. In particular, we differentiate between two distinct cases. The first case assumes that the sensor nodes are equipped with receivers and, hence, every node can exploit the wireless link to distribute its information to its neighbors. We then devise an efficient deterministic cooperation strategy where the neighboring nodes act as virtual antennas in a beamforming configuration. The second, and more challenging, scenario restricts the capability of sensor nodes to transmit only. In this case, we argue that statistical cooperative source-channel coding techniques still yield significant performance gains in certain relevant scenarios. Specifically, we propose a low complexity cooperative source-channel coding scheme based on the proper use of low-density generator matrix codes. This scheme is shown to outperform the recently proposed joint source-channel coding scheme (Garcia-Frias et al., 2002) in the case of highly correlated sources. In both the deterministic and statistical cooperation scenarios, we develop analytical results that guide the optimization of the proposed schemes and validate the performance gains observed in simulations.
\end{abstract}

Index Terms-Beamforming, cooperative transmission schemes, correlated sources, dense sensor networks, Gaussian multiple-access channel (GMAC), low-density generator matrix (LDGM) codes, source-channel coding.

\section{INTRODUCTION}

$\mathbf{T}$ HE INTEREST in wireless sensor networks has been rising sharply in recent years. One of the basic challenges in such networks is the reliable transmission of the correlated observations available at the different sensors to one, or more, collector nodes. This gives rise to the so called many-to-one or reach-back channel in the case of one collector node [1], [4], [7], [9].

Works on constructing coding schemes for this scenario have focused primarily on the problem of distributed compression of correlated sources. In particular, for the case of discrete sources considered in this paper, recent works have proposed coding

Manuscript received July 15, 2003; revised February 1, 2004. This work was supported in part by the National Science Foundation under Grant CCR-0118859 and Grant ITR-0219892. This paper was presented in part at the 41 st Annual Allerton Conference on Communication, Control, and Computing, Monticello, IL, October 2003.

The authors are with the Department of Electrical Engineering, The Ohio State University, Columbus, OH 43210 USA (e-mail: helgamal@ece.osu.edu). Digital Object Identifier 10.1109/JSAC.2004.830889 schemes that approach the Slepian-Wolf fundamental limit on the achievable compression rates (e.g., [8], [10], and [11]). The main limitation of these works is the assumption of dummy noiseless channels between the set of sensors and the collector node. This assumption leads, therefore, to the separate optimization of source and channel coding. While this separation is well motivated for the point-to-point case, as shown by Shannon, it can entail significant performance losses in more general scenarios [2], [3]. As shown in the sequel, the many-to-one channel considered here is one of the instances where the separation of source and channel coding entails significant performance losses (for a certain range of parameters).

This nonoptimality of separation-based schemes in sensor networks motivates the fresh look, proposed here, at the design of cooperative source-channel coding schemes that effectively exploit the wireless medium. We use the term cooperative source-channel coding to differentiate our schemes from available joint source-channel coding techniques inspired by the separation principle (e.g., [5], [6], and [12]). We further differentiate between two distinct scenarios of cooperation. The first one assumes that the sensor nodes are equipped with receivers which allows an arbitrary sensor node to distribute its observations to its neighbors. We then devise an efficient deterministic cooperation strategy where the neighboring nodes act as virtual antennas in a beamforming configuration. As shown in the sequel, Slepian-Wolf coding plays an integral role in minimizing the amount of resources used to facilitate intersensor communication here.

The second scenario considers the more challenging task of facilitating node cooperation without relying on intersensor communication. Specifically, this scenario corresponds to the transmission of correlated sources over a Gaussian multiple-access channel (GMAC) [3]. Here, we devise a low complexity blind cooperation scheme based on the simultaneous transmission of low-density generator matrix (LDGM) codes. As shown later, the low density of the generator matrix of LDGM codes is an important ingredient of the proposed scheme. In a nutshell, the two cooperation schemes strive to map the correlation between the source observations into correlation between the transmitted signals. In doing this, we follow the information theoretic guidelines offered in [2]-[4] for maximizing the capacity of the many-to-one channel. To the best of our knowledge, this is the first attempt to construct explicit coding schemes for statistical cooperation.

In both the deterministic and statistical cooperation scenarios, we develop analytical results that guide the optimization of the proposed schemes and validate the performance gains observed in simulations. Aided by analytical and simulation results, our 
schemes are shown to outperform the joint source-channel coding scheme recently proposed by Garcia-Frias et al. [6]. The performance gains offered by the proposed blind cooperative scheme demonstrate the strict suboptimality of schemes based on source/channel separation in certain instances of the many-to-one channel. Although we focus mainly on the two-source scenario in the majority of the paper, the extension of the proposed schemes to dense sensor networks [4], [9] is briefly discussed.

The rest of the paper is organized as follows. In Section II, we introduce the system model along with our notation. We present the deterministic cooperation scheme in Section III where we also analyze its performance limits. In Section IV, we construct the statistical cooperation scheme for source-channel coding and develop an approximate analytical tool for optimizing the different parameters involved in this scheme. In Section V, we present representative numerical results demonstrating the performance gains offered by the proposed schemes. Finally, Section VI offers some concluding remarks.

\section{SYSTEM MODEL AND NOTATION}

Unless otherwise stated, we consider two correlated binary sources $\mathbf{U}$ and $\mathbf{V}$. We denote the sequence generated by source $\mathbf{U}$ as $\left\{u_{1}, u_{2}, \ldots\right\}$ and that of source $\mathbf{V}$ as $\left\{v_{1}, v_{2}, \ldots\right\}$. We further assume that the data generated by each of the two sources is independent identically distributed (i.i.d.). The correlation between the two sources is determined by $\operatorname{Pr}\left(u_{i} \neq v_{i}\right)=$ $p, \forall i$. We assume that the sources share a wireless additive white Gaussian noise (AWGN) channel. Code constructions that account for the effects of multipath fading will be considered in future works.

For simplicity of presentation, we use a discrete time real model where $y[j]$, the real signal received at the collector node at time $j$, is given by

$$
y[j]=x_{u}[j]+x_{v}[j]+n[j]
$$

where $x_{u}[j]$ and $x_{v}[j]$ are the symbols transmitted at time $j$ by $\mathbf{U}$ and $\mathbf{V}$, respectively, and $n[j]$ is the zero-mean real Gaussian noise sample at time $j$ with variance $\sigma_{c}^{2}$. We impose only a constraint on the total average power, i.e.,

$$
E\left\{\left|x_{u}\right|^{2}+\left|x_{v}\right|^{2}\right\} \leq P_{\text {avg }} .
$$

In (1), it is assumed that the source nodes are synchronized with a common clock. This requirement is important since beamforming plays a key role in the proposed schemes as detailed later. For the intersensor communication link, we also adopt the same AWGN channel model and refer to the variance of the noise impairing this link as $\sigma_{u v}^{2}$. The difference in noise variances corresponds to the difference in quality between the two links (equivalently, one can model the difference in quality by allowing for different attenuation factors). We define $k$ to be the number of bits generated by each sensor during one time slot and $N$ to be the number of symbols transmitted in one time slot (i.e., $j \in\{1, \ldots, N\}$ ). The total rate of transmission is, therefore, given by

$$
R=\frac{k H(\mathbf{U}, \mathbf{V})}{N}
$$

where $H(\mathbf{U}, \mathbf{V})$ refers to the joint entropy of the two sources.

As a theoretical benchmark, we consider the separation-based scheme consisting of distributed Slepian-Wolf source coding of the two sources followed by optimal channel coding for the GMAC. Here, we observe that with only the sum-power constraint, time-division multiple access (TDMA) achieves the capacity of the GMAC [2]. It follows that the fundamental limit on the performance of the separation-based scheme is given by the following equivalent relations [2]:

$$
\begin{aligned}
\frac{k}{N} H(\mathbf{U}, \mathbf{V}) & =R \leq \frac{1}{2} \log \left(1+\frac{P_{\mathrm{avg}}}{\sigma_{c}^{2}}\right) \\
P_{\mathrm{avg}} & \geq \sigma_{c}^{2}\left(2^{2 R}-1\right) .
\end{aligned}
$$

We note that this separation-based scheme is blind in the sense that it does not require intersensor communication. This scheme also does not need the synchronization assumption required for the proposed cooperative strategies. As a practical benchmark, we consider the recently proposed scheme in [6]. This scheme employs punctured turbo codes for joint sourcechannel coding by the individual sources followed by a TDMA strategy. At the receiver, joint iterative decoding is used to recover the two sources. Though this scheme is called a joint source-channel coding scheme, one can see that it is inspired by the separation principle (as highlighted by the use of TDMA) and joint coding and decoding only serve to narrow the gap with the theoretical limit given in (5).

\section{INFORMED (DETERMINISTIC) COOPERATION}

In this section, we propose a novel cooperation strategy that exploits the wireless channel to facilitate intersensor communication. The proposed strategy is tailored for applications where the distance between the sensors is much smaller than the distance between the set of sensor nodes and the collector node. In our model, this assumption translates to $\sigma_{u v}^{2} / \sigma_{c}^{2} \ll 1$. We remark that this assumption, in general, holds for the class of dense sensor networks considered in [4] and [9].

Our scheme utilizes a cooperative TDMA strategy in which a fraction $f_{1}$ of the time slot is devoted to intersensor communication. The time allocated for intersensor communication is divided equally among the two sensors such that at a particular instant of time, only one sensor transmits while the other sensor listens. This exchange of information between the sensors allows them to cooperate deterministically by transmitting simultaneously in a beamforming configuration to the collector node for the remaining fraction $\left(1-f_{1}\right)$ of time. The optimization of the parameter $f_{1}$ is considered in the sequel. A schematic diagram of the proposed scheme is shown in Fig. 1.

Let us consider the transmission of information from source $\mathbf{U}$ to source $\mathbf{V}$. This is the classical example of source coding with side information where one needs only to transmit the information corresponding to the conditional entropy $H(\mathbf{U} \mid \mathbf{V})$ [11]. Here, we use a regular low-density parity check (LDPC) coding scheme, where we encode the information bits of $\mathbf{U}$ using a $\left(k, N_{1}\right)$ systematic LDPC code. We then puncture all the systematic bits and transmit only the $r_{1}=\left(N_{1}-k\right)$ parity 


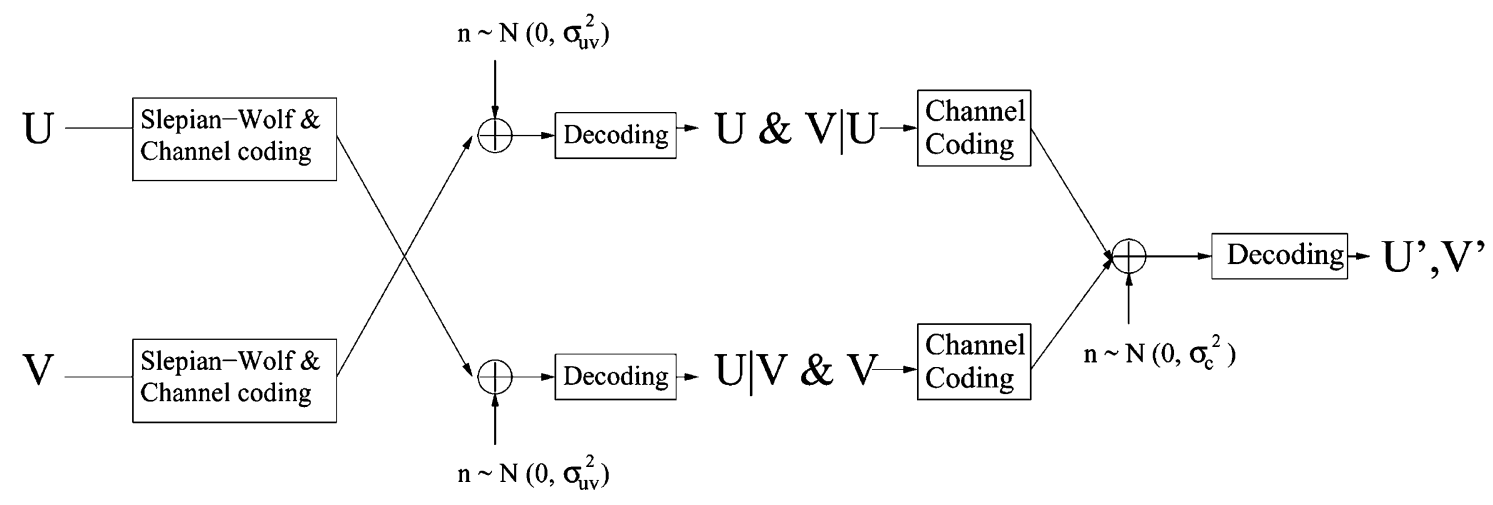

Fig. 1. Schematic diagram of the informed cooperation scheme.

bits through the channel. The number of parity bits $r_{1}$ of the LDPC code is related to the fraction $f_{1}$ through the relation

$$
r_{1}=\frac{N f_{1} \log _{2}|\Omega|}{2}
$$

where $|\Omega|$ is the cardinality of the constellation used for intersensor communication. The same scheme is used for transmission from source $\mathbf{V}$ to source $\mathbf{U}$. It is straightforward to see that this scheme allows for a direct application of the Turbo principle in the decoder where the correlation between the two sources is used as prior information (e.g., [5] and [6]). The details of the decoder are omitted here for brevity.

Assuming successful decoding in the first stage, the two sensor nodes now know both $\mathbf{U}$ and $\mathbf{V}$ and hence can cooperate in delivering the information to the collector node. Through the simultaneous transmission of identical signals in this stage, one can exploit the $3-\mathrm{dB}$ beamforming gain. In this stage, we again employ a similar LDPC coding/decoding scheme. In particular, we first transmit $\mathbf{U}$ to the collector node using a systematic LDPC code. Now, the transmission of $\mathbf{V}$ to the collector node again reduces to the problem of source coding with side information discussed above. It is interesting to note that the intersensor communication in the first stage reduces the many-to-one problem to a point-to-point communication problem, for which the source/channel separation theorem holds.

To further enhance the performance, we allow for using different power levels in the two transmission stages (i.e., during intersensor communication, the active source transmits at a power of $P_{u v}$, whereas during the second stage each source transmits at a power equal to $P_{c} / 2$ ). We use the information theoretic analysis in the following section to guide the choice of $f_{1}, P_{c}$, and $P_{u v}$. We realize that further performance gains can be reaped by using more sophisticated irregular LDPC codes. In this paper, we did not pursue this direction since it amounts to a straightforward modification of the proposed techniques and, as such, does not contribute to additional insights. The proposed scheme may also be improved by allowing the collector node to exploit the received information during the intersensor communication stage (observe that in wireless channels, this information is delivered to the collector node for free). In our work, no attempt was made to exploit this received information in order to minimize the decoder complexity and facilitate the theoretical analysis presented in the following section.

\section{A. Information Theoretical Analysis}

In this section, we compute the information theoretic limit that bounds the performance of the proposed scheme. We further use this analysis to find the information theoretic optimal values of $f_{1}, P_{c}$, and $P_{u v}$, and characterize the scenarios where the proposed cooperative TDMA scheme outperforms techniques based on the separate optimization of source and channel coding. We note that the optimality implied by our analysis only holds when capacity-achieving codes are used and need not necessarily carry over to practical implementations. However, the optimal values obtained using the capacity achieving codes serve as good approximations to those obtained for LDPC codes.

From the previous discussion of the proposed cooperative TDMA scheme, one can see that successful intersensor communication is possible only if ${ }^{1}$ [2]

$$
\begin{aligned}
\frac{k H(\mathbf{U} \mid \mathbf{V})}{N} & =\frac{k H(p)}{N} \\
& \leq\left(\frac{f_{1}}{2}\right) \frac{1}{2} \log \left(1+\frac{P_{u v}}{\sigma_{u v}^{2}}\right)
\end{aligned}
$$

Hence, the power $P_{u v}$ required for intersensor communication is lower bounded by

$$
P_{u v} \geq \sigma_{u v}^{2}\left(2^{\frac{4 k H(p)}{N f_{1}}}-1\right) .
$$

Similarly, the communication to the collector node will be successful only if

$$
\begin{aligned}
\frac{k H(\mathbf{U}, \mathbf{V})}{N} & =\frac{k(1+H(p))}{N} \\
& \leq\left(1-f_{1}\right) \frac{1}{2} \log \left(1+\frac{2 P_{c}}{\sigma_{c}^{2}}\right)
\end{aligned}
$$

The factor of 2 in the $\log$ term is due to beamforming (since each source transmits with power $\left(P_{c} / 2\right)$, and the signals add coherently, the received power is $\left.2 P_{c}\right)$. Hence, the total power $P_{c}$ allocated for communication with the collector node should satisfy

$$
P_{c} \geq \frac{\sigma_{c}^{2}}{2}\left(2^{\frac{2 k(1+H(p))}{N\left(1-f_{1}\right)}}-1\right) .
$$

\footnotetext{
${ }^{1}$ This capacity can be achieved only for Gaussian inputs but it serves as a good approximation of the achievable rate for other constellations at low signal-tonoise ratio (SNR).
} 
The value $f_{1}^{*}$ that minimizes the average power

$$
P_{\text {avg }}=P_{u v}\left(f_{1}\right)+P_{c}\left(1-f_{1}\right)
$$

can be found by numerically solving the following equation:

$$
\begin{gathered}
\left(1-\frac{2 \sigma_{u v}^{2}}{\sigma_{c}^{2}}\right)+\left(\frac{2 \sigma_{u v}^{2}}{\sigma_{c}^{2}}\right) 2^{\frac{4 k H(p)}{N f_{1}^{*}}}\left(1-\frac{4 k H(p) \ln (2)}{N f_{1}^{*}}\right) \\
+2^{\frac{2 k(1+H(p))}{N\left(1-f_{1}^{*}\right)}}\left(\frac{2 k(1+H(p)) \ln (2)}{N\left(1-f_{1}^{*}\right)}-1\right)=0 .
\end{gathered}
$$

The minimum power levels $P_{u v}^{*}$ and $P_{c}^{*}$ corresponding to $f_{1}^{*}$ can be obtained by imposing equality in (7) and (8), respectively. It is interesting to note that these optimal power levels not only depend on the correlation between the sensors, but also on the quality of the intersensor communication channel.

One can now argue for the asymptotic optimality of the proposed scheme as follows. In the case of $p \rightarrow 0$ and/or $\sigma_{u v}^{2} / \sigma_{c}^{2} \rightarrow$ 0 , one can see that the proper fraction of time assigned for intersensor communication will be $f_{1}^{*} \rightarrow 0$. This means that the proposed scheme will achieve the maximum $3-\mathrm{dB}$ gain over separation-based schemes [this 3-dB gain can be seen by letting $f_{1}=0$ in (8) and (9), and comparing the result with (5)].

\section{B. Generalization to Dense Sensor Networks}

We now extend the deterministic cooperation scheme to the scenario of dense sensor networks with $n$ nodes. The correlated random sequences corresponding to the observations of the $n$ sensors will be referred to as $\mathbf{U}_{\mathbf{1}}, \mathbf{U}_{\mathbf{2}}, \ldots, \mathbf{U}_{\mathbf{n}}$. For a particular realization, the output of sensor $\mathbf{U}_{\mathbf{i}}$ is denoted by $\mathbf{u}_{\mathbf{i}}=\left\{u_{i, 1}, \ldots, u_{i, k}\right\}$. We assume the following correlation model between the outputs of the sensor nodes. The output of the first source is denoted as $\mathbf{u}_{\mathbf{1}}=\mathbf{e}_{\mathbf{1}}$, where $\mathbf{e}_{\mathbf{1}}$ is an i.i.d. sequence with $\operatorname{Pr}\left(e_{1, l}=0\right)=\operatorname{Pr}\left(e_{1, l}=1\right)=(1 / 2), \forall l$. The output of source $\mathbf{U}_{\mathbf{i}}$ is given with respect to the output of source $\mathbf{U}_{\mathbf{i}-\mathbf{1}}$ as $\mathbf{u}_{\mathbf{i}}=\mathbf{u}_{\mathbf{i}-\mathbf{1}} \oplus \mathbf{e}_{\mathbf{i}}$, where $\mathbf{e}_{\mathbf{i}}(i \in\{2, \ldots, n\})$ is an i.i.d.sequence with $\operatorname{Pr}\left(e_{i, l}=1\right)=(p / n), \operatorname{Pr}\left(e_{i, l}=0\right)=(1-(p / n)), \forall l$. This assignment ensures that the correlation between adjacent sensors increases as the number of sensors " $n$ " increases. The correlation parameter between the streams $\mathbf{U}_{\mathbf{i}}$ and $\mathbf{U}_{\mathbf{j}}$ is denoted by $p_{i, j}$. We adopt an AWGN channel model for the intersensor links. We denote the noise variance of the channel between the sensors $\mathbf{U}_{\mathbf{i}}$ and $\mathbf{U}_{\mathbf{j}}$ by $\sigma_{i j}^{2}$. We assume that these noise variances follow the model $\sigma_{i j}^{2}=((|i-j| / n))^{\gamma}$, where $\gamma>0$. These models for the correlation and the intersensor channels are intended to correspond to the case where the geographical area of the network is fixed ${ }^{2}$ and, hence, increasing the number of sensors results in an increase in the density. This increase in density, subsequently, results in a higher correlation between the data streams generated at adjacent nodes and also a better channel between adjacent sensors. Here, we fix the transmission rate and characterize the scaling law of the minimum average power $P_{\text {avg }}^{*}$ required by the cooperation scheme as the number of sensors grows (i.e., as $n \rightarrow \infty$ ). The gain offered by the proposed scheme will be illustrated by comparing this scaling law with the corresponding one in the separation-based scheme.

In the proposed scheme, we divide the $n$ sensors into groups of $n^{\alpha}$ each, where $0<\alpha<1$. As before, we adopt a TDMA

\footnotetext{
${ }^{2} \mathrm{Here}$, we have chosen a one-dimensional model to simplify the analysis.
}

strategy wherein time slots are allocated to the different groups periodically. Within a fraction $f_{1}$ of their allocated time slot, the sensors in the designated group exchange their information. This exchange of information allows the sensors within the group to cooperate deterministically by transmitting simultaneously in a beamforming configuration to the collector node for the remaining fraction $\left(1-f_{1}\right)$ of their time slot. As before, we allow for using different power levels in the two transmission stages (i.e., during intersensor communication, the active source $\mathbf{U}_{\mathbf{i}}$ transmits at a power of $P_{i}$, whereas during communication with the collector node, each source within the group transmits at a power of $\left.\left(P_{c} / n^{\alpha}\right)\right)$.

Without loss of generality, we consider the transmission of the first group of sensor nodes $\mathbf{U}_{1}, \mathbf{U}_{2}, \ldots, \mathbf{U}_{\mathbf{n}^{\alpha}}$. The effective transmission rate is given by

$$
R=\frac{k H\left(\mathbf{U}_{\mathbf{1}}, \mathbf{U}_{\mathbf{2}}, \ldots, \mathbf{U}_{\mathbf{n}^{\alpha}}\right)}{N} .
$$

During intersensor communication, each sensor in the group is allocated a fraction $\left(\left(f_{1} / n^{\alpha}\right)\right)$ of the time slot. During this fraction, the designated sensor node must provide enough information to the other members of the group such that all these other sensors can recover its observation. To minimize complexity, we propose a suboptimal strategy where the designated sensor node sends only one stream with enough rate such that the sensor with the least correlated observation in the group can recover the observations of the transmitting sensor (assuming successful decoding of the transmitted stream). Using the standard random binning argument, one can see that all the sensors in the group can also recover the observations of the transmitting sensor assuming correct decoding of this stream. To ensure successful decoding by all receiving sensors, enough transmit power should be allocated such that the receiving sensor which experiences the worst channel can decode successfully. These two arguments imply the following limit on the proposed intersensor communication strategy (assuming the use of capacity-achieving channel codes)

$$
\frac{k H\left(\hat{p}_{i}\right)}{N} \leq\left(\frac{f_{1}}{n^{\alpha}}\right) \frac{1}{2} \log \left(1+\frac{P_{i}}{\hat{\sigma}_{i}^{2}}\right), \quad \forall i \in\left\{1, \ldots, n^{\alpha}\right\}
$$

or equivalently

$$
P_{i} \geq \hat{\sigma}_{i}^{2}\left(2^{\frac{2 k n^{\alpha} H\left(\hat{p}_{i}\right)}{N f_{1}}}-1\right), \quad \forall i \in\left\{1, \ldots, n^{\alpha}\right\}
$$

where

$$
\hat{\sigma}_{i}^{2}=\max _{j \in\left\{1, \ldots, n^{\alpha}\right\} \backslash\{i\}} \sigma_{i j}^{2} \quad \text { and } \quad \hat{p}_{i}=\max _{j \in\left\{1, \ldots, n^{\alpha}\right\} \backslash\{i\}} p_{i, j} .
$$

At the end of the intersensor communication stage, all the sensors in the group know all the corresponding observations and, hence, can cooperate deterministically. The communication to the collector node will then be successful if (again assuming the use of capacity-achieving codes)

$$
\frac{k H\left(\mathbf{U}_{\mathbf{1}}, \mathbf{U}_{\mathbf{2}}, \ldots, \mathbf{U}_{\mathbf{n}^{\alpha}}\right)}{N} \leq\left(1-f_{1}\right) \frac{1}{2} \log \left(1+\frac{n^{\alpha} P_{c}}{\sigma_{c}^{2}}\right)
$$

or equivalently

$$
P_{c} \geq \frac{\sigma_{c}^{2}}{n^{\alpha}}\left(2^{\frac{2 k H\left(\mathrm{U}_{1}, \mathrm{U}_{2}, \ldots, \mathrm{U}_{\mathrm{n}} \alpha\right)}{N\left(1-f_{1}\right)}}-1\right) .
$$


The minimum required average power $P_{\text {avg }}^{*}$ is, hence, given by

$$
P_{\text {avg }}^{*}=\frac{f_{1}^{*}}{n^{\alpha}}\left(P_{1}^{*}+P_{2}^{*}+\cdots+P_{n^{\alpha}}^{*}\right)+\left(1-f_{1}^{*}\right) P_{c}^{*}
$$

which yields

$$
\begin{aligned}
P_{\mathrm{avg}}^{*}=\left(\frac{\left(1-f_{1}^{*}\right) \sigma_{c}^{2}}{n^{\alpha}}\right) & \left(2^{\frac{2 k H\left(\mathrm{U}_{1}, \mathrm{U}_{2}, \ldots, \mathrm{U}_{\mathrm{n}} \boldsymbol{\alpha}\right)}{N\left(1-f_{1}^{*}\right)}}-1\right) \\
& +\frac{f_{1}^{*}}{n^{\alpha}} \sum_{i=1}^{n^{\alpha}} \hat{\sigma}_{i}^{2}\left(2^{\frac{2 k n^{\alpha} H\left(\hat{p}_{i}\right)}{N f_{1}^{*}}}-1\right) .
\end{aligned}
$$

From the assumptions on the correlation model and the intersensor channel, one can easily see that

$$
\hat{\sigma}_{i}^{2} \leq\left(\frac{n^{\alpha}}{n}\right)^{\gamma}=\frac{1}{n^{(1-\alpha) \gamma}} \quad \text { and } \quad H\left(\hat{p}_{i}\right) \leq H\left(p_{1, n^{\alpha}}\right)
$$

$\forall i \in\left\{1, \ldots, n^{\alpha}\right\}$. Combining these upper bounds with (13) yields the following upper bound on the minimum required average power

$$
\begin{aligned}
P_{\text {avg }}^{*} \leq & \left(\frac{\left(1-f_{1}^{*}\right) \sigma_{c}^{2}}{n^{\alpha}}\right)\left(2^{\frac{2 R}{\left(1-f_{1}^{*}\right)}}-1\right) \\
& \quad+\left(\frac{f_{1}^{*}}{n^{(1-\alpha) \gamma}}\right)\left(2^{\frac{2 R n^{\alpha} H\left(p_{1, n} \alpha\right)}{H\left(\mathrm{U}_{\mathbf{1}}, \mathrm{U}_{\mathbf{2}}, \ldots, \mathrm{U}_{\mathbf{n}} \boldsymbol{\alpha}\right) f_{1}^{*}}}-1\right) .
\end{aligned}
$$

For large $n$, we have

$$
\begin{gathered}
\frac{n^{\alpha} H\left(p_{1, n^{\alpha}}\right)}{H\left(\mathbf{U}_{\mathbf{1}}, \mathbf{U}_{\mathbf{2}}, \ldots, \mathbf{U}_{\mathbf{n}^{\alpha}}\right)} \leq \frac{n^{\alpha} H\left(\frac{n^{\alpha} p}{n}\right)}{1+\left(n^{\alpha}-1\right) H\left(\frac{p}{n}\right)} \\
\leq \frac{\delta p}{n^{(1-2 \alpha)}} \leq \delta p, \quad \text { if } \alpha<\frac{1}{2}
\end{gathered}
$$

where $\delta$ is chosen such that $H(q)<\delta q$ for all $q<p$. Using this in (14), we get [for large $n$ and $\alpha<(1 / 2)$ ]

$$
\begin{aligned}
P_{\text {avg }}^{*} \leq\left(\frac{\left(1-f_{1}^{*}\right) \sigma_{c}^{2}}{n^{\alpha}}\right) & \left(2^{\frac{2 R}{\left(1-f_{1}^{*}\right)}}-1\right) \\
& +\left(\frac{f_{1}^{*}}{n^{(1-\alpha) \gamma}}\right)\left(2^{\frac{2 R \delta p}{f_{1}^{*}}}-1\right) .
\end{aligned}
$$

It is now evident from (15) that for a fixed and finite rate $R$, the required average transmission power $P_{\text {avg }}^{*}$ of the proposed scheme scales as

$$
P_{\text {avg }}^{*} \leq O\left(\frac{1}{n^{\min \{\alpha,(1-\alpha) \gamma\}}}\right), \quad \text { with } \alpha<\frac{1}{2}
$$

as the number of sensor nodes $n$ grows to infinity. ${ }^{3}$ From (4), it can be shown that $P_{\text {avg }}^{*}=O(1)$ for the separation-based scheme as $n \rightarrow \infty$ and the transmission rate is fixed. Comparing these two scaling laws, one can see the significant power savings offered by the deterministic cooperation scheme in dense sensor networks.

\section{Blind (STATISTICAL) COOPERATION}

In this section, we consider the more challenging scenario where the sensor nodes are restricted to transmit only. This constraint only allows the sensors to cooperate statistically. Similar

\footnotetext{
${ }^{3}$ We observe that this result is also valid for more general correlation models than the one assumed here.
}

to Section III, we first focus on the case of two sensor nodes and then briefly outline the extension of the proposed scheme to dense sensor networks with large number of nodes. In fact, this is the well-known multiple-access channel with correlated sources problem which has been studied in [3] and the proposed cooperation scheme attempts to capitalize on the information theoretic insights offered in [2] and [3].

More precisely, our scheme is inspired by the following insightful observation from [2] "To maximize the capacity of the GMAC, one should preserve the correlation between the inputs of the channel. Slepian-Wolf encoding, on the other hand, gets rid of the correlation." The simplest way to illustrate the idea is to consider, again, the asymptotic case with $p=0$. In this case, the separation-based scheme would involve transmission from only one source (say $\mathbf{U}$ ) since the other source does not generate any novel information (i.e., $H(\mathbf{V} \mid \mathbf{U})=0$ ). The optimal cooperative scheme, however, is to transmit the identically encoded streams from both sources simultaneously (the correlation coefficient between the two transmitted signals is one). One can easily see that, in this toy example, the optimal cooperative scheme will attain the beamforming 3 - $\mathrm{dB}$ gain over the separation-based scheme. In fact, the main objective of the deterministic cooperation scheme proposed earlier is to facilitate this beamforming for arbitrary $p \neq 0$ by exploiting the intersensor communications capability. In the current blind scenario, a different solution for handling $p \neq 0$ is needed.

To simplify the presentation and analysis, we restrict ourselves to binary phase-shift keying (BPSK) modulation in this section. The proposed scheme (for arbitrary $p$ ) relies on the simultaneous transmission of two identical systematic LDGM codes, as shown in Fig. 2, to facilitate statistical cooperation. The generator matrix of the LDGM code can be represented as

$$
\mathbf{G}=\left(\mathbf{I}_{\mathbf{k} \times \mathbf{k}} \mid \mathbf{G}^{\prime}\right)
$$

where $\mathbf{G}^{\prime}$ is a regular sparse matrix with $d_{v}$ ones in each row and $d_{c}$ ones in each column. First, it is easy to see that the correlation between the transmitted signals is preserved in the systematic part of the code word. The low density of the generator matrix of LDGM codes is intended to map the correlation between the two observed streams into correlation between the two parity sequences as argued next. The $\xi$ th parity bit for the first source (i.e., $p_{\xi}^{u}$ ) is given by

$$
p_{\xi}^{u}=u_{\xi_{1}} \oplus u_{\xi_{2}} \oplus \cdots \oplus u_{\xi_{d_{c}}}
$$

where $\left\{u_{\xi_{\ell}}: \ell=1, \ldots, d_{c}\right\}$ are the information bits corresponding to ones in the $\xi$ th column of $\mathbf{G}^{\prime}$. If $p_{\xi}^{u}$ and $p_{\xi}^{v}$ are the $\xi$ th parity bits of sources $\mathbf{U}$ and $\mathbf{V}$, respectively, then

$$
\operatorname{Pr}\left(p_{\xi}^{u} \neq p_{\xi}^{v}\right)=\frac{1-(1-2 p)^{d_{c}}}{2} \approx d_{c} p, \quad \text { for } p \ll 1 .
$$

So by choosing small values for $d_{c}$, one can increase the correlation between the parity sequences. On the other hand, it is well known that decreasing $d_{c}$ degrades the performance of the stand-alone LDGM code (e.g., [6]). Therefore, one would expect the existence of a value for $d_{c}$, which strikes the optimal tradeoff between these two goals for every value of $p$. Experimentally, we have found $d_{c}=4$ to achieve near optimal 


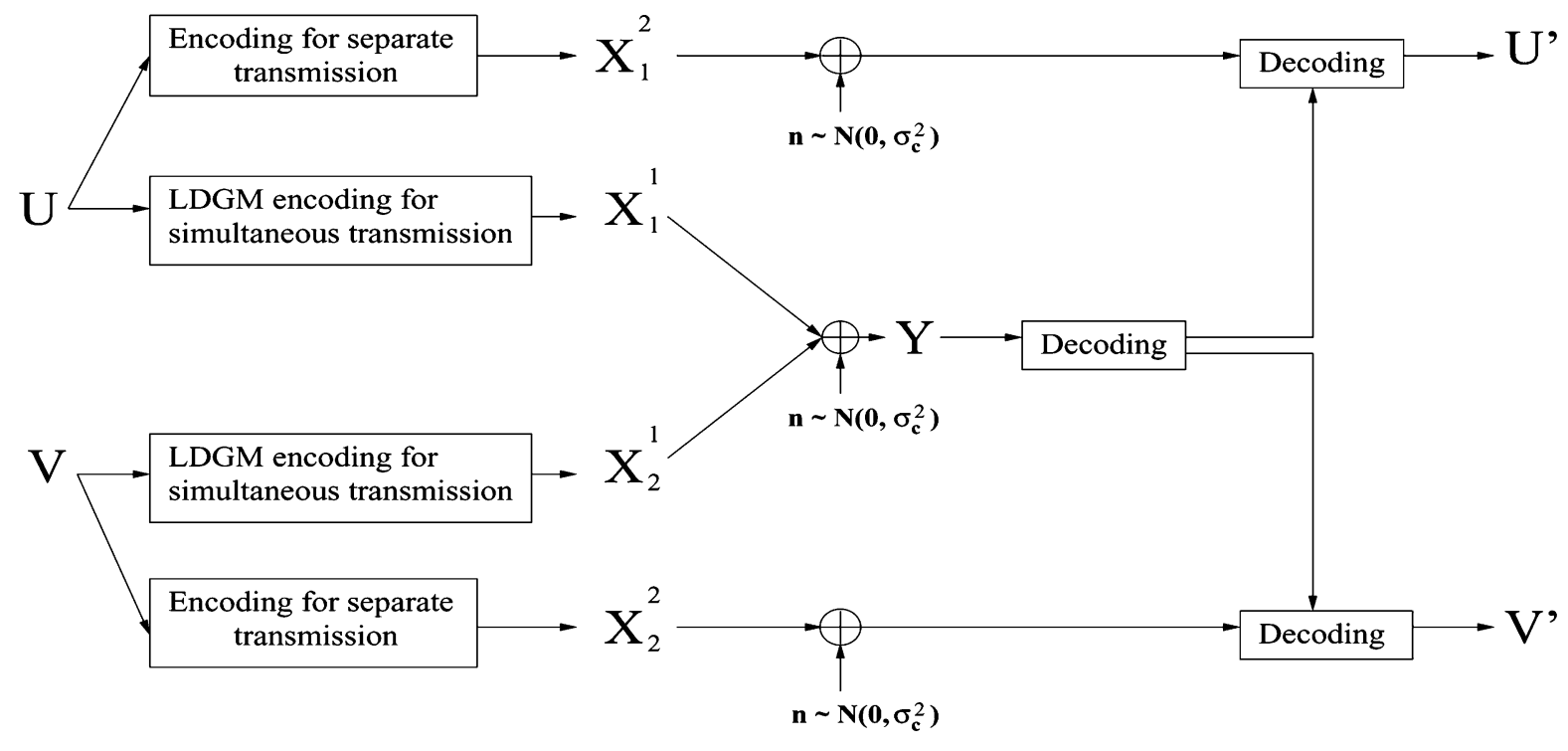

Fig. 2. Schematic diagram of the blind cooperation scheme.

performance for the values of $p$ of interest (highly correlated sources).

Unfortunately, the simultaneous transmission stage by itself is not sufficient for successful decoding, as demonstrated by the following argument. Consider the simultaneous transmission of the systematic bits. If $u_{l} \neq v_{l}$, then the corresponding received signal at the $l$ th time instant is just the Gaussian noise. Moreover, it is easy to see that, for small values of $p$, all the check equations in which $u_{l}$ and $v_{l}$ participate will result in different parity bits for the two encoded streams and, hence, the corresponding received signals will again be just Gaussian noise. This gives rise to decoder ambiguity in those bits (i.e., the probability that $u_{l}=1, v_{l}=0$ is the same as $u_{l}=0, v_{l}=1$ ). To resolve this ambiguity, we require both sources to send additional parity bits separately (i.e., we use a TDMA protocol, where every source is assigned an equal interval to send its unique parity sequence). We refer to this part of the code word as the separate transmission to differentiate it from the earlier simultaneous transmission part. Since in this separate transmission part, the sources do not benefit from the beamforming gain, we allow for a different power allocation. In the simultaneous transmission part, each source transmits at a power of $\left(P_{c} / 2\right)$, whereas during separate transmission, the active source transmits at a power of $m P_{c}$. It is now clear that there are several parameters to be optimized in the proposed scheme [e.g., power allocation (i.e., $m$ ), the connectivity of the generator matrix, and the ratio of the simultaneous transmission part length to the separate part length]. In the following section, we develop an approximate analytical tool for guiding the optimization of these different parameters. Finally, we note that ambiguity bits are a feature of our scheme that resulted from our attempt to maximize the cooperative (beamforming) gain in the simultaneous transmission stage when the symbols are identical (i.e., using identical codes and equal power for the two sources). In the proposed scheme, we resolved these ambiguity bits through the separate transmission stage. More fundamentally, one can argue that there is a tradeoff between the cooperative gain, when the transmitted symbols are identical, and the cancellation loss, when the symbols are different. The door is still open for constructing schemes that realize the optimal tradeoff between these two effects.

We conclude the discussion of the proposed scheme by giving a brief description of the decoding strategy. To minimize complexity, we adopt a two-stage decoding strategy. In the first stage, the received signal during the simultaneous transmission phase is used to decode the bits in which $\mathbf{U}$ and $\mathbf{V}$ are identical. For iterative decoding, the log-likelihood ratios (LLRs) are initialized using the rule

$$
q_{j, 0}=\frac{-4 \sqrt{\frac{P_{c}}{2}} y[j]}{\sigma_{c}^{2}} .
$$

One can see that this suboptimal initialization rule ignores the presence of erased symbols, and hence, the iterative decoder only attempts to identify the bits in which $u_{l}=v_{l}$. In the second stage, decoding is done separately for each source. Iterative decoding in this stage utilizes the LLR values obtained from the first stage of decoding and the LLR values of the additional parity bits that are transmitted separately by the sources. As expected, this stage strives to identify the ambiguous bits that are ignored in the first stage. It is important to observe that this decoder does not exploit the a priori information about the correlation between the two sources. We realize that more sophisticated approaches, that exploit this information, can be employed. We, however, restricted our attention to this approach to minimize the decoder complexity. The independence of the decoding procedure on the correlation between the two sources is also desirable in certain applications that require robust decoding algorithms.

\section{A. Approximate Analysis}

Here, we develop an approximate analytical tool for optimizing the different parameters that govern the performance of the proposed blind cooperation scheme. In addition, this tool offers approximate estimates of the fundamental limits of the 
proposed scheme. It should be noted that the analysis presented here will be mainly used to optimize the performance of the proposed blind cooperation scheme, and does not give the achievable rate for the GMAC with correlated sources.

Analogous to Section III, we let $N_{s}=N\left(1-f_{1}\right)$ be the number of bits transmitted simultaneously, and $r_{1}=\left(N f_{1} / 2\right)$ be the number of additional parity bits transmitted by each source separately. Although the two sources transmit $N_{s}$ bits simultaneously, the first-stage decoder only attempts to identify the bits for which $u_{l}=v_{l}$ from only the un-erased received symbols. Hence, the effective rate of the code used in the first stage is $R_{s}=(k(1-p)) /\left(N_{s}\left(1-p^{\prime}\right)\right)$, where $p^{\prime}=\left(k p+\left(N_{s}-k\right) d_{c} p\right) /\left(N_{s}\right)$ is the average erasure probability (the averaging takes into account the fact that the erasure probability is different in the systematic and nonsystematic parts). Noting that the received SNR in the simultaneous transmission part is $\left(2 P_{c} / \sigma_{c}^{2}\right)$, we obtain the following approximate condition for successful decoding:

$$
\frac{k(1-p)}{N_{s}(1-p \prime)} \approx \frac{1}{2} \log \left(1+\frac{2 P_{c}}{\sigma_{c}^{2}}\right) .
$$

After the first decoding stage, the resulting $k$-bit sequences of the two sources will contain $(k p / 2)$ errors each (assuming we flip a coin in the erased positions). Alternatively, one can treat those unidentified bits as erasures and obtain slightly more optimistic results. Our experimental results, however, show that modeling those bits as errors gives more accurate predictions. For successful decoding in the second stage, we have

$$
k H\left(\frac{p}{2}\right) \approx \frac{r_{1}}{2} \log \left(1+\frac{m P_{c}}{\sigma_{c}^{2}}\right) .
$$

From (17) and the relation $\left(k / N_{s}\right)=\left(d_{c} /\left(d_{v}+d_{c}\right)\right)$, we get

$$
\begin{gathered}
\left(\frac{d_{c}}{d_{c}+d_{v}}\right)\left(\frac{1-p}{1-p d_{c}\left(\frac{1+d_{v}}{d_{c}+d_{v}}\right)}\right) \approx \frac{1}{2} \log \left(1+\frac{2 P_{c}}{\sigma_{c}^{2}}\right) \\
\Rightarrow d_{v} \approx \frac{\left(\frac{d_{c}(1-p)}{\frac{1}{2} \log \left(1+\frac{2 P_{c}}{\sigma_{c}^{2}}\right)}\right)-d_{c}(1-p)}{1-p d_{c}} .
\end{gathered}
$$

Using the fact that $(k / N)=(k) /\left(N_{s}+2 r_{1}\right)$, we get

$$
\frac{k}{r_{1}}=\frac{2}{\frac{N}{k}-\left(\frac{d_{v}+d_{c}}{d_{c}}\right)} \text {. }
$$

From (18) and (20)

$$
\left(\frac{2}{\frac{N}{k}-\left(\frac{d_{v}+d_{c}}{d_{c}}\right)}\right) H\left(\frac{p}{2}\right) \approx \frac{1}{2} \log \left(1+\frac{m P_{c}}{\sigma_{c}^{2}}\right) .
$$

For a particular value of $P_{c}$, the value of $d_{v}$ can be obtained from (19). Also, from the known value of $(k / N), m$ can now be found from (21). One can then obtain the average power $P_{\text {avg }}$ as

$$
P_{\mathrm{avg}}=P_{c}\left(\frac{N_{s}+2 r_{1} m}{N}\right)
$$

The optimal value $P_{c}^{*}$ is the one which minimizes the average power for given $k, N$, and $p$. The corresponding optimal values of $d_{v}$ and $m$ can be obtained from (19) and (21), respectively. Here, we find these optimal values numerically.

\section{B. Generalization to Dense Sensor Networks}

We now extend the blind cooperation scheme to the scenario of dense sensor networks with $n$ nodes. We adopt the same correlation model used in Section III-B. It is worth noting that there is no need here to invoke any path loss model for the intersensor channels since intersensor communication is assumed to be infeasible. Analogous to Section III-B, our goal is to characterize the scaling law of the minimum average power required by the blind cooperation scheme as the number of sensors grows to infinity.

Again, we divide the $n$ sensors into groups of $n^{\alpha}$ each, where $0<\alpha<1$. The sensors within each group cooperate statistically to transmit their information to the collector node. Within each group, the sensors first transmit simultaneously using systematic LDGM codes, and then each sensor transmits additional information separately to resolve the ambiguity of the unidentified bits. Since we are primarily interested in finding a lower bound on the efficiency of the proposed approach, the total transmission power (i.e., $P_{c}$ ) is assumed to be the same for both simultaneous and separate transmissions. Thus, the output of each sensor is divided into blocks of $k$ bits each, and each block is encoded to a codeword of $N_{s}$ bits. These codewords are then transmitted simultaneously by all the sensors within the group. Let $p_{e}$ be the probability of error for each source after decoding in the first stage. Now, each source has to transmit $k H\left(p_{e}\right)$ bits of information separately to the collector node. These $k H\left(p_{e}\right)$ bits are encoded into a codeword of $r_{1}$ bits. Again inspired by the desire to obtain a lower bound, we assume that during simultaneous transmission only those bits which are the same for all the sensors within the group will be decoded correctly and the rest are considered as errors.

Let the encoded vector of source $i$ be given by $\mathbf{x}_{\mathbf{i}}(i \in$ $\left.\left\{1, \ldots, n^{\alpha}\right\}\right)$. Then, the encoded outputs of source $(i-1)$ and source $i$ are related by $\mathbf{x}_{\mathbf{i}}=\mathbf{x}_{\mathbf{i}-\mathbf{1}} \oplus \mathbf{g}_{\mathbf{i}}$, where $\mathbf{g}_{\mathbf{i}}\left(i \in\left\{2, \ldots, n^{\alpha}\right\}\right)$ is an $N_{s}$-length sequence with $\operatorname{Pr}\left(g_{i, l}=1\right) \leq\left(d_{c} p / n\right) \forall l$. To simplify notation, we let $p_{1}=d_{c} p$. Thus, the encoded sequence $\mathbf{x}_{\mathbf{i}}$ can be expressed as

$$
\mathbf{x}_{\mathbf{i}}=\mathbf{x}_{\mathbf{1}} \oplus \mathbf{g}_{\mathbf{2}} \oplus \mathbf{g}_{\mathbf{3}} \oplus \cdots \oplus \mathbf{g}_{\mathbf{i}}
$$

By letting $\mathbf{f}_{\mathbf{i}}=\sum_{j=2}^{i} \mathbf{g}_{\mathbf{j}}$, we get $\mathbf{x}_{\mathbf{i}}=\mathbf{x}_{\mathbf{1}} \oplus \mathbf{f}_{\mathbf{i}}$. Now, using the union bound, the probability $\operatorname{Pr}\left(f_{i, l}=1\right)$ can be upper bounded as

$$
\operatorname{Pr}\left(f_{i, l}=1\right) \leq \sum_{j=2}^{i} \operatorname{Pr}\left(g_{j, l}=1\right) \leq \frac{(i-1) p_{1}}{n} .
$$

Let $p_{c}$ be the probability that the $l$ th bit is the same for all $\mathbf{u}_{\mathbf{i}}$ in the group, which occurs when $e_{i, l}=0$ for $i \in\left\{2, \ldots, n^{\alpha}\right\}$, and similarly, $p_{c_{1}}$ be the probability that the $l$ th bit is the same for all $\mathbf{x}_{\mathbf{i}}$, which occurs when $g_{i, l}=0$ for $i \in\left\{2, \ldots, n^{\alpha}\right\}$.

Thus

$$
p_{c}=\left(1-\frac{p}{n}\right)^{\left(n^{\alpha}-1\right)} \quad \text { and } \quad p_{c_{1}} \geq\left(1-\frac{p_{1}}{n}\right)^{\left(n^{\alpha}-1\right)} \text {. }
$$


Since the suboptimal decoder used in our scheme for simultaneous transmission attempts to decode only those bits for which all the source outputs are the same, the effective code rate (with respect to the bits for which all $g_{i, l}$ 's are zero) is $\left(k p_{c} / N_{s} p_{c_{1}}\right)$. One can then argue that successful decoding for the common information is possible only if

$$
\frac{k p_{c}}{N_{s} p_{c_{1}}} \leq \frac{1}{2} \log \left(1+\frac{n^{\alpha} P_{c}}{\sigma_{c}^{2}}\right)
$$

For separate transmission, the corresponding condition is given by

$$
\frac{k H\left(p_{e}\right)}{r_{1}} \leq \frac{1}{2} \log \left(1+\frac{P_{c}}{\sigma_{c}^{2}}\right)
$$

The effective rate of transmission is

$$
R=\frac{k\left(1+\left(n^{\alpha}-1\right) H\left(\frac{p}{n}\right)\right)}{N} .
$$

From (26) and (27), we have

$$
\begin{aligned}
\frac{N}{k} & =\frac{N_{s}}{k}+\frac{n^{\alpha} r_{1}}{k} \\
& =\frac{\beta}{\frac{1}{2} \log \left(1+\frac{n^{\alpha} P_{c}}{\sigma_{c}^{2}}\right)}+\frac{n^{\alpha} H\left(p_{e}\right)}{\frac{1}{2} \log \left(1+\frac{P_{c}}{\sigma_{c}^{2}}\right)}
\end{aligned}
$$

where $\beta=\left(p_{c} / p_{c_{1}}\right)$. Since we are concerned with the fundamental performance limit, the inequalities in (26) and (27) are taken as equalities in (29).

The relation between the transmission power $P_{c}$ and the rate of transmission $R$ can be obtained from (28) and (29) as

$$
R=\frac{1+\left(n^{\alpha}-1\right) H\left(\frac{p}{n}\right)}{\left(\frac{\beta}{\frac{1}{2} \log \left(1+\frac{n^{\alpha} P_{c}}{\sigma_{c}^{2}}\right)}+\frac{n^{\alpha} H\left(p_{e}\right)}{\frac{1}{2} \log \left(1+\frac{P_{c}}{\sigma_{c}^{2}}\right)}\right)} .
$$

Now, we wish to characterize the scaling law of the power gain allowed by statistical cooperation for any fixed, and finite, transmission rate $R$ as $n \rightarrow \infty$. A first step is to see (using standard arguments) that $\beta=\left(p_{c} / p_{c_{1}}\right) \rightarrow 1$ as $n \rightarrow \infty$.

Next, we need to find $p_{e}$ (or an upper bound on $p_{e}$ ) and its scaling with respect to $n$. Since successful decoding occurs only when the bits transmitted from all the sources are the same (i.e., when all $f_{i, l}$ 's are 0 ), we can use the union bound to get the following upper bound on $p_{e}$ :

$$
\begin{aligned}
p_{e} & =\operatorname{Pr}\left(\text { at least one } f_{i}=1\right) \leq \sum_{i=2}^{n^{\alpha}} \frac{(i-1) p_{1}}{n} \\
& \Rightarrow p_{e} \leq \frac{n^{\alpha}\left(n^{\alpha}-1\right) p_{1}}{2 n} \leq \frac{n^{2 \alpha} p_{1}}{n} .
\end{aligned}
$$

Now, consider the term $n^{\alpha} H\left(p_{e}\right)$. This can be upper bounded by

$$
n^{\alpha} H\left(p_{e}\right)<\gamma n^{\alpha} p_{e} \leq \gamma \frac{p_{1}}{n^{(1-3 \alpha)}}
$$

where $\gamma$ is chosen such that $H(q)<\gamma q$ for all $q<p_{1}$. Then, $n^{\alpha} H\left(p_{e}\right) \rightarrow 0$ for large $n$ and $\alpha<(1 / 3)$.
From (30) and (32), we have for large values of $n$

$$
R\left(\frac{1}{\frac{1}{2} \log \left(1+\frac{n^{\alpha} P_{c}}{\sigma_{c}^{2}}\right)}\right)=1, \quad \text { for } \alpha<\frac{1}{3} .
$$

Therefore, for a fixed rate $R$, the minimum average transmission power $P_{\text {avg }}^{*}$ can be expressed as

$$
P_{\mathrm{avg}}^{*}=P_{c}=\frac{\sigma_{c}^{2}\left(2^{2 R}-1\right)}{n^{\alpha}}
$$

for large $n$ if $\alpha<(1 / 3)$. Hence, for a fixed rate of transmission $R$, the required transmission power $P_{\text {avg }}^{*}$ of the proposed scheme scales as $P_{\mathrm{avg}}^{*}=O\left(1 /\left(n^{(1 / 3)-\epsilon}\right)\right)$ if the number of nodes in a group is chosen to be $n^{(1 / 3)-\epsilon}$. Similar to the deterministic cooperation scheme, one can now see the significant savings in power offered by the proposed statistical cooperation scheme, compared with the separation-based scheme, in dense sensor networks.

\section{Numerical Results}

In this section, we present numerical results quantifying the performance of the proposed cooperation schemes in certain representative scenarios. Throughout this section, we restrict ourselves to the case with only two sensor nodes. In our simulations, we choose $k=9500$ and $N=40000$ as in [6]. The bit-error rate was averaged over 1000 frames per source. The simulation threshold is defined as the value of $P_{\text {avg }} / \sigma_{c}^{2}$ corresponding to a bit error rate of $10^{-5}$.

\section{A. Informed Cooperation}

1) Analytical Results: Using the analytical steps sketched in Section III-A, the average transmitted power $P_{\text {avg }}$ can be found for a given fraction $f_{1}$. The values of $P_{\text {avg }}$ for different values of $f_{1}$ are reported in Fig. 3 for different values of $\sigma_{u v}^{2} / \sigma_{c}^{2}$ and $p=0.01$. As is evident from the figure, the deterministic cooperation scheme performs better than the separation-based scheme even when the noise variance of the intersensor channel is comparable with that of the channel between the sensors and the receiver (i.e., $\sigma_{u v}^{2} / \sigma_{c}^{2}=1$ ).

A detailed comparison of the theoretical limits $\left(P_{\text {avg }}^{*} / \sigma_{c}^{2}\right)$ for the proposed informed cooperation scheme and the separation-based scheme is provided in Table I. It is evident from the table that the proposed cooperative scheme always performs better than the separation scheme, irrespective of the value of the correlation parameter $p$, when the intersensor channel is very good (i.e., $\sigma_{u v}^{2} / \sigma_{c}^{2} \leq 0.1$ ).

2) Simulation Results: We used LDPC codes with $d_{v}=3$ throughout our simulations. For intersensor communication, a $(9500,13500)$ LDPC code was used and only the 4000 parity bits were modulated and transmitted through the channel. The modulation scheme used for intersensor communication was 16-PAM with Gray mapping. The motivation of using a higher order constellation here is to exploit the better quality of the intersensor channel. For communication with the collector node, we used a $(9500,28500)$ LDPC code for the transmission of $\mathbf{U}$. After $\mathbf{U}$ is decoded at the collector node, we used a (9500, 


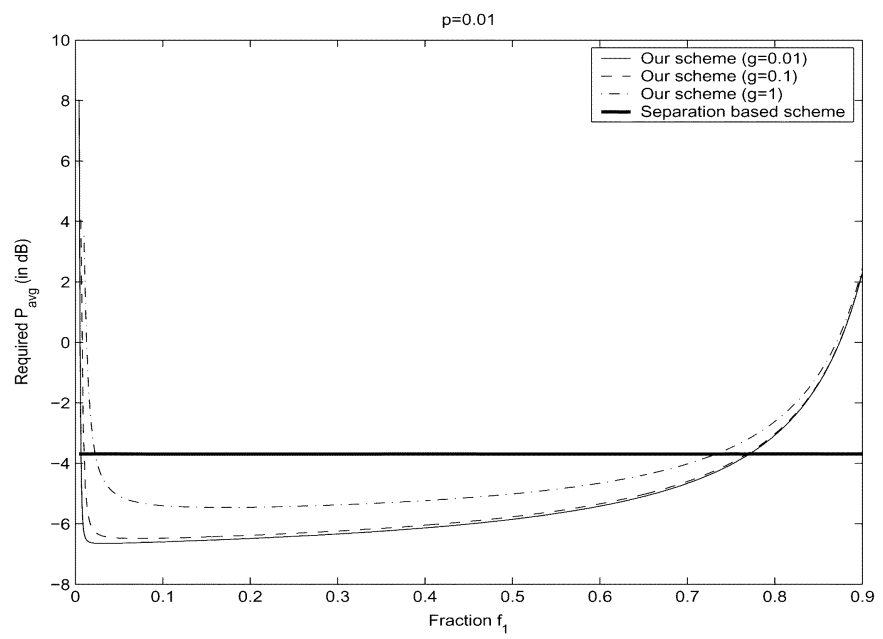

Fig. 3. Theoretical limits of the informed cooperation scheme and the separation-based scheme for different values of $g=\sigma_{u v}^{2} / \sigma_{c}^{2}$ for $p=0.01$.

TABLE I

COMPARISON OF THE INFORMED COOPERATION SCHEME AND THE SEPARATION-BASED SCHEME (THEORETICAL LIMITS)

\begin{tabular}{c|c|c|c|c|c|c}
\hline \multirow{2}{*}{$p$} & \multirow{3}{*}{$*$} & \multirow{2}{*}{$\begin{array}{c}\text { Separation } \\
\text { scheme }\end{array}$} & \multicolumn{4}{|c}{ Informed Cooperation scheme } \\
\cline { 4 - 7 } & & Threshold & \multicolumn{2}{|c|}{$\sigma_{u v}^{2} / \sigma_{c}^{2}=0.01$} & \multicolumn{2}{|c}{$\sigma_{u v}^{2} / \sigma_{c}^{2}=0.1$} \\
\cline { 4 - 7 } & & Threshold & Gain & Threshold & Gain \\
\hline \hline & & & & & & \\
0.5 & 0.475 & $-0.31 \mathrm{~dB}$ & $-2.53 \mathrm{~dB}$ & $2.22 \mathrm{~dB}$ & $-1.17 \mathrm{~dB}$ & $0.86 \mathrm{~dB}$ \\
0.2 & 0.41 & $-1.18 \mathrm{~dB}$ & $-3.65 \mathrm{~dB}$ & $2.47 \mathrm{~dB}$ & $-2.58 \mathrm{~dB}$ & $1.40 \mathrm{~dB}$ \\
0.1 & 0.35 & $-2.06 \mathrm{~dB}$ & $-4.74 \mathrm{~dB}$ & $2.68 \mathrm{~dB}$ & $-3.97 \mathrm{~dB}$ & $1.91 \mathrm{~dB}$ \\
0.05 & 0.31 & $-2.78 \mathrm{~dB}$ & $-5.59 \mathrm{~dB}$ & $2.81 \mathrm{~dB}$ & $-5.08 \mathrm{~dB}$ & $2.30 \mathrm{~dB}$ \\
0.025 & 0.28 & $-3.29 \mathrm{~dB}$ & $-6.18 \mathrm{~dB}$ & $2.89 \mathrm{~dB}$ & $-5.86 \mathrm{~dB}$ & $2.57 \mathrm{~dB}$ \\
0.01 & 0.26 & $-3.69 \mathrm{~dB}$ & $-6.65 \mathrm{~dB}$ & $2.96 \mathrm{~dB}$ & $-6.48 \mathrm{~dB}$ & $2.79 \mathrm{~dB}$ \\
\hline
\end{tabular}

19000) LDPC code for the transmission of $\mathbf{V}$ and transmitted only the 9500 parity bits through the channel.

For $p=0.01$, our simulation results show that when $\sigma_{u v}^{2} / \sigma_{c}^{2}=0.01$, the threshold value of the informed cooperation scheme is $-4.33 \mathrm{~dB}$, which is about $2.32 \mathrm{~dB}$ away from the theoretical limit, and shows an improvement of $2.61 \mathrm{~dB}$ over the coding scheme proposed by Garcia-Frias , Zhong, and Zhao (GZZ) in [6]. ${ }^{4}$ The informed cooperation scheme also shows an improvement of $0.35 \mathrm{~dB}$ over the blind cooperation scheme reported in the next subsection. For $p=0.025$, the threshold value of the informed cooperation scheme when $\sigma_{u v}^{2} / \sigma_{c}^{2}=0.01$ is $-3.92 \mathrm{~dB}$, which is about $2.26 \mathrm{~dB}$ away from the theoretical limit and offers an improvement of $2.36 \mathrm{~dB}$ over GZZ scheme [6]. The informed cooperation scheme also offers an improvement of $1.1 \mathrm{~dB}$ over the blind cooperation scheme. As expected, when $\sigma_{u v}^{2} / \sigma_{c}^{2}=0.1$ there is a decrease in the gain obtained by the informed cooperation scheme over GZZ scheme. The results are summarized in Table II.

\footnotetext{
${ }^{4}$ To the best of the authors' knowledge, the performance of this scheme represents the current state of the art for this scenario.
}

TABLE II

COMPARISON OF THE INFORMED COOPERATION SCHEME AND GZZ SCHEME (SIMULATION RESULTS)

\begin{tabular}{|c|c|c|c|c|c|c|}
\hline \multirow[b]{2}{*}{$\frac{\sigma_{u v}^{2}}{\sigma_{c}^{2}}$} & \multirow[b]{2}{*}{$p$} & \multirow[b]{2}{*}{$R$} & \multicolumn{2}{|c|}{$\begin{array}{l}\text { Informed Cooperation } \\
\text { scheme }\end{array}$} & \multirow{2}{*}{$\begin{array}{c}\text { GZZ } \\
\text { scheme } \\
\text { Threshold } \\
\text { (sim) }\end{array}$} & \multirow[b]{2}{*}{ Gain } \\
\hline & & & $\begin{array}{l}\text { Threshold } \\
\text { (theory) }\end{array}$ & $\begin{array}{c}\text { Threshold } \\
\text { (sim) }\end{array}$ & & \\
\hline 0.01 & 0.025 & 0.28 & $-6.18 \mathrm{~dB}$ & $-3.92 \mathrm{~dB}$ & $-1.56 \mathrm{~dB}$ & $2.36 \mathrm{~dB}$ \\
\hline 0.01 & 0.01 & 0.26 & $-6.65 \mathrm{~dB}$ & $-4.33 \mathrm{~dB}$ & $-1.72 \mathrm{~dB}$ & $2.61 \mathrm{~dB}$ \\
\hline 0.1 & 0.025 & 0.28 & $-5.86 \mathrm{~dB}$ & $-2.81 \mathrm{~dB}$ & $-1.56 \mathrm{~dB}$ & $1.25 \mathrm{~dB}$ \\
\hline 0.1 & 0.01 & 0.26 & $-6.48 \mathrm{~dB}$ & $-3.99 \mathrm{~dB}$ & $-1.72 \mathrm{~dB}$ & $2.27 \mathrm{~dB}$ \\
\hline
\end{tabular}

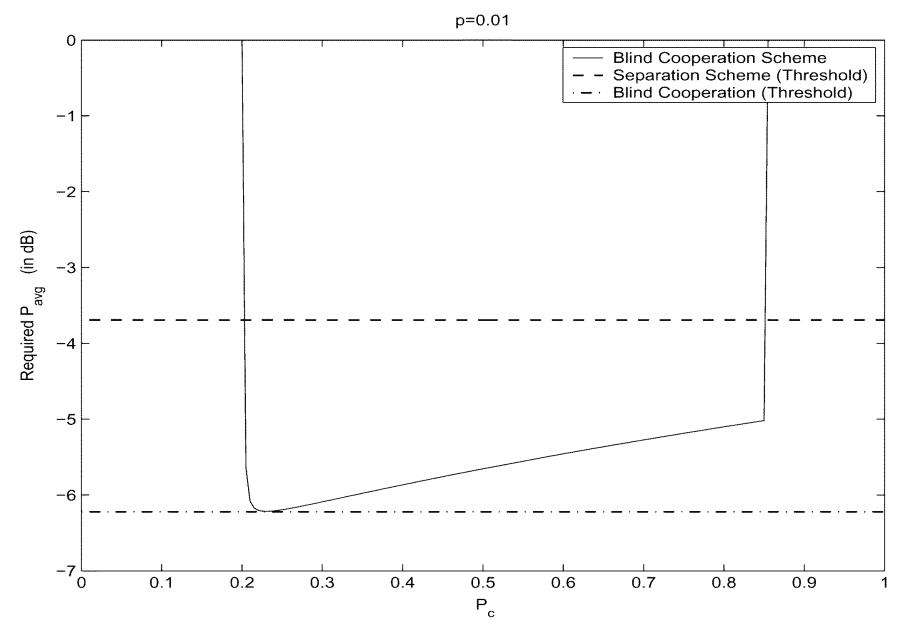

Fig. 4. Comparison of the analytical performance of the proposed blind cooperation scheme and the separation-based scheme for $p=0.01$.

\section{B. Blind Cooperation}

1) Analytical Results: From the analytical steps illustrated in Section IV-A, the average transmitted power $P_{\text {avg }}$ can be found for a given power $P_{c}$ allocated for the simultaneous transmission stage. The values of $P_{\text {avg }}$ for different values of $P_{c}$ are plotted in Fig. 4 for $p=0.01$. From the figure, it is clear that there exists a value of $P_{c}$ (and corresponding values of $d_{v}$ and $m)$ for which $P_{\text {avg }}$ is minimized. The proposed scheme performs best at this point, and the analytical threshold $P_{\mathrm{avg}}^{*} / \sigma_{c}^{2}$ is computed using this value.

A detailed comparison of the theoretical limits for the proposed blind cooperation scheme and the separation-based scheme is given in Table III for different values of $p$.

2) Simulation Results: We used an LDGM code with $d_{c}=4$ for the simultaneous transmission part. We have found experimentally that this value for $d_{c}$ strikes a near optimal tradeoff. For the separate transmission part, an LDGM code with $\left(d_{v}, d_{c}\right)=$ $(31,50)$ is used throughout our experiments. For $p=0.01$, the approximate analysis in Section IV-A predicts that the best performance of the proposed scheme is achieved with $d_{v} \approx$ 11 and $m=1.36$. Experimentally, we found $d_{v}=8$ and $m=1.25$ to achieve optimal performance. Using these parameters, the threshold value of the proposed scheme is $-3.98 \mathrm{~dB}$, 
TABLE III

COMPARISON OF THE BLIND COOPERATION SCHEME AND THE SEPARATION-BASED SCHEME (THEORETICAL LIMITS)

\begin{tabular}{c|c|c|c|c}
\hline$p$ & $R$ & $\begin{array}{c}\text { Separation } \\
\text { scheme } \\
\text { Threshold } \\
\text { (theory) }\end{array}$ & $\begin{array}{c}\text { Blind Cooperation } \\
\text { scheme } \\
\text { Threshold } \\
\text { (theory) }\end{array}$ & Gain \\
\hline \hline & & & & \\
0.168 & 0.39 & $-1.4 \mathrm{~dB}$ & $-1.4 \mathrm{~dB}$ & $0 \mathrm{~dB}$ \\
0.15 & 0.38 & $-1.55 \mathrm{~dB}$ & $-1.7 \mathrm{~dB}$ & $0.15 \mathrm{~dB}$ \\
0.1 & 0.35 & $-2.06 \mathrm{~dB}$ & $-2.72 \mathrm{~dB}$ & $0.66 \mathrm{~dB}$ \\
0.05 & 0.31 & $-2.78 \mathrm{~dB}$ & $-4.24 \mathrm{~dB}$ & $1.46 \mathrm{~dB}$ \\
0.025 & 0.28 & $-3.28 \mathrm{~dB}$ & $-5.32 \mathrm{~dB}$ & $2.05 \mathrm{~dB}$ \\
0.01 & 0.26 & $-3.69 \mathrm{~dB}$ & $-6.21 \mathrm{~dB}$ & $2.52 \mathrm{~dB}$ \\
\hline
\end{tabular}

TABLE IV

COMPARISON OF THE BLIND COOPERATION SCHEME AND GZZ SCHEME (SIMULATION RESULTS)

\begin{tabular}{|c|c|c|c|c|c|c|}
\hline \multirow[t]{2}{*}{$p$} & \multirow[t]{2}{*}{$R$} & \multicolumn{3}{|c|}{$\begin{array}{c}\text { Blind Cooperation } \\
\text { scheme }\end{array}$} & \multirow{2}{*}{$\begin{array}{c}\text { GZZ } \\
\text { scheme } \\
\text { Threshold } \\
\text { (sim) }\end{array}$} & \multirow[t]{2}{*}{ Gain } \\
\hline & & $\begin{array}{l}\text { Threshold } \\
\text { (theory) }\end{array}$ & $\begin{array}{c}\text { Threshold } \\
\text { (sim) }\end{array}$ & Gap & & \\
\hline 0.025 & 0.28 & $-5.32 \mathrm{~dB}$ & $-2.82 \mathrm{~dB}$ & $2.5 \mathrm{~dB}$ & $-1.56 \mathrm{~dB}$ & $1.26 \mathrm{~dB}$ \\
\hline 0.01 & 0.26 & $-6.21 \mathrm{~dB}$ & $-3.98 \mathrm{~dB}$ & $2.23 \mathrm{~dB}$ & $-1.72 \mathrm{~dB}$ & $2.26 \mathrm{~dB}$ \\
\hline
\end{tabular}

which is about $2.23 \mathrm{~dB}$ away from the theoretical limit predicted by our approximate analysis, and offers an improvement of $2.26 \mathrm{~dB}$ over GZZ coding scheme [6]. We note that the simulation threshold of the proposed scheme is better than the theoretical limit of separation-based schemes in this case $(0.29-\mathrm{dB}$ gain). This is a strong evidence supporting our claim on the gain possible through cooperation. For $p=0.025$, the theoretical analysis predicts the optimal values of $d_{v} \approx 9$ and $m=$ 1.32 , while experimentally optimal performance was achieved with $d_{v}=8$ and $m=1.15$. The threshold value of the proposed scheme is $-2.82 \mathrm{~dB}$, which is about $2.5 \mathrm{~dB}$ away from the approximate theoretical limit and shows an improvement of $1.26 \mathrm{~dB}$ over GZZ scheme [6]. The results are summarized in Table IV.

It is clear that as $p$ increases, the number of bits which are erased in the simultaneous transmission part increases, and hence, the performance of the proposed scheme degrades. One would, therefore, expect the existence of a threshold value for $p$ at which the separation-based scheme starts to outperform the proposed scheme. Our theoretical analysis predicts that this threshold is $p=0.168$ for this set of system parameters. Experimentally, however, we have found GZZ scheme to outperform the proposed blind cooperation scheme for $p>0.05$. Finally, we observe that the gap between theoretical and simulation results, in both the deterministic and statistical cooperation scenarios, is expected. One can further work toward minimizing the gap by constructing more powerful component codes, increasing the block length, and employing more sophisticated decoding algorithms. Our results, however, still serve the purpose of highlighting the power of cooperative source-channel coding schemes.

\section{CONCLUDING REMARKS}

In this paper, we proposed novel cooperative source-channel coding techniques for the transmission of correlated sources over wireless channels. The proposed techniques utilize the correlation between the sources to maximize the capacity of the many-to-one channel. When possible, we exploit intersensor communication to facilitate deterministic cooperation between the sources. When intersensor communication is prohibited, we devised a statistical cooperation scheme for source-channel coding. The proposed scheme is based on the simultaneous transmission of identical LDGM codes from the sources to the collector node. Throughout the paper, we guided our design with information theoretic insights and analysis. Finally, numerical results were presented to establish the gain allowed by the proposed schemes over schemes inspired by the separation principle in certain representative scenarios. Our objective in this work was mainly to demonstrate the power of cooperative source-channel coding techniques that efficiently exploit the correlation between the sources. We hope that this paper will motivate further work in this exciting area. For example, the design of optimal component codes for the statistical cooperation strategy is an interesting open problem. The LDGM codes used here strike a very desirable balance between preserving the correlation between the transmitted signals and the power of the stand-alone code but, in our view, further research can potentially yield more powerful codes.

\section{REFERENCES}

[1] J. Barros and S. Servetto, "On the capacity of the reachback channel in wireless sensor networks," in Proc. IEEE Workshop Multimedia Signal Processing, 2002, pp. 408-411.

[2] T. Cover and J. Thomas, Elements of Information Theory. New York: Wiley, 1991.

[3] T. M. Cover, A. A. E. Gamal, and M. Salehi, "Multiple-access channels with arbitrarily correlated sources," IEEE Trans. Inform. Theory, vol. 26, pp. 648-657, Nov. 1980.

[4] H. E. Gamal, "On the scaling laws of dense wireless sensor networks," IEEE Trans. Inform. Theory, Apr. 2003, submitted for publication.

[5] J. Garcia-Frias, "Joint source-channel decoding of correlated sources over noisy channels," in Proc. IEEE Data Compression Conf., Mar. 2001, pp. 283-292.

[6] J. Garcia-Frias, W. Zhong, and Y. Zhao, "Iterative decoding schemes for source and joint source-channel coding of correlated sources," in Proc. Conf. Rec. 36th Asilomar Conf. Signals, Systems, Computers, Nov. 2002, pp. $250-256$

[7] P. Gupta and P. Kumar, "The capacity of wireless networks," IEEE Trans. Inform. Theory, vol. 46, pp. 388-404, Mar. 2000.

[8] A. Liveris, Z. Xiong, and C. Georghiades, "Compression of binary sources with side information using low-density parity-check codes," in Proc. Global Telecommunications Conf., vol. 2, Nov. 2002, pp. 1300-1304.

[9] D. Marco, E. J. Duarte-Melo, M. Liu, and D. L. Neuhoff, "On the many-to-one transport capacity of a dense wireless sensor network and the compressibility of its data," in Proc. Int. Workshop Information Processing in Sensor Networks, Apr. 2003, pp. 1-16.

[10] S. Pradhan and K. Ramchandran, "Distributed source coding: Symmetric rates and applications to sensor networks," in Proc. IEEE Data Compression Conf., Mar. 2000, pp. 363-372.

[11] D. Slepian and J. Wolf, "Noiseless coding of correlated information sources," IEEE Trans. Inform. Theory, vol. 19, pp. 471-480, July 1973.

[12] W. Zhong, Y. Zhao, and J. Garcia-Frias, "Turbo-like codes for distributed joint source-channel coding of correlated senders in multiple access channels," Proc. Conf. Rec. 37th Asilomar Conf. Signals, Systems, Computers, pp. 840-844, Nov. 2003. 


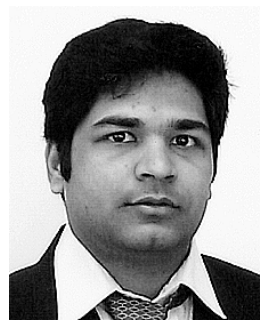

Arul D. Murugan received the B.Tech. degree in electrical engineering from the Indian Institute of Technology, Madras, India, in 2001. He is currently working toward the Ph.D. degree in electrical engineering at The Ohio State University (OSU), Columbus.

His current research interests include sourcechannel coding and performance of sensor networks.

Mr. Murugan is a recipient of the OSU Distinguished University Fellowship (2001).

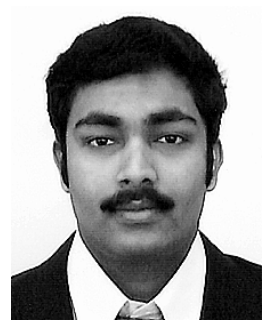

Praveen K. Gopala received the B.E. degree in electronics and communication engineering from the College of Engineering, Guindy, Anna University, Madras, India, in 2002. He is currently working toward the Ph.D. degree in electrical engineering at The Ohio State University (OSU), Columbus.

His current research interests include the evaluation of the fundamental limits of cooperative protocols for sensor networks and the design of novel protocols that achieve those limits.

Mr. Gopala is a recipient of the OSU University

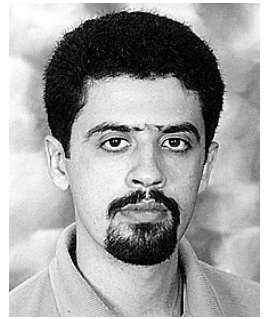

Hesham El Gamal (M'99-SM'03) received the B.S. and M.S. degrees in electrical engineering from Cairo University, Cairo, Egypt, in 1993 and 1996, respectively, and the Ph.D. degree in electrical and computer engineering from the University of Maryland, College Park, in 1999.

From 1993 to 1996, he served as a Project Manager in the Middle East Regional Office of Alcatel Telecom. From 1996 to 1999, he was a Research Assistant in the Department of Electrical and Computer Engineering, University of Maryland. From February 1999 to December 2000, he was with the Advanced Development Group, Hughes Network Systems (HNS), Germantown, MD, as a Senior Member of the Technical Staff. In the Fall of 1999, he served as a Lecturer at the University of Maryland. Since January 2001, he has been an Assistant Professor in the Electrical Engineering Department, The Ohio State University (OSU), Columbus. He held visiting appointments at the University of California, Los Angeles (UCLA) (Fall 2002, Winter 2003) and the Institut Eurecom (Summer 2003). He is the holder of four patents with nine patent applications pending.

Dr. El Gamal is a recipient of the HNS Annual Achievement Award (2000), the OSU College of Engineering Lumley Research Award (2003), the OSU Electrical Engineering Department FARMER Young Faculty Development Fund (2003-2008), and the National Science Foundation CAREER Award (2004). He is an Associate Editor for Space-Time Coding and Spread Spectrum for the IEEE TRANSACTIONS ON COMMUNICATIONS. 